\title{
Super-tough artificial nacre based on graphene oxide via synergistic interface interactions of $\pi-\pi$ stacking and hydrogen bonding
}

Pingan Song ${ }^{\mathrm{a}, \mathrm{b}, \mathrm{c}, *}$, Zhiguang $\mathrm{Xu}^{\mathrm{b}}{ }^{\S}$, Yuanpeng $\mathrm{Wu}^{\mathrm{d}}$, Qunfeng Cheng ${ }^{\mathrm{e}, *}$, Qipeng Guo ${ }^{\mathrm{b}, *}$, and Hao Wang ${ }^{\mathrm{c}}$

${ }^{a}$ Department of Materials, College of Engineering, Zhejiang A \& F University, Hangzhou, Zhejiang, 311300, China

${ }^{b}$ Polymers Research Group, Institute for Frontier Materials, Deakin University, Locked Bag 20000, Geelong, Victoria 3220, Australia

${ }^{c}$ Centre for Future Materials, University of Southern Queensland, Toowoomba, Queensland, 4350, Australia

${ }^{d}$ School of Materials Science and Engineering, Southwest Petroleum University, Chengdu, Sichuan, 610500, China, ${ }^{e}$ Key Laboratory of Bio-inspired Smart Interfacial Science and Technology of Ministry of Education, School of Chemistry Environment, Beihang University, Beijing 100191, China

${ }^{\S} \mathrm{P}$. Song and Z. Xu contributed equally to the work.

ABSTRACT: Inspired by interfacial interactions of protein matrix and the crystal platelets in nacre, herein, asuper-tough artificial nacre was produced through constructing the synergistic interface interactions of $\pi-\pi$ interaction and hydrogen bonding between graphene oxide (GO) nanosheets and sulfonated styrene-ethylene/butylene-styrene copolymer synthesized with multifunctional benzene. The resultant GO-based artificial

* Correspondence should be addressed: +86 571 63743976. E-mail: pingansong@gmail.com (P. Song), cheng@buaa.edu.cn (Q. Cheng), and qguo@deakin.edu.au (Q. Guo) 
nacre showed super-high toughness of $15.3 \pm 2.5 \mathrm{MJ} / \mathrm{m}^{3}$, superior to natural nacre and other GO-based nanocomposites. The ultra-tough property of the novel nacre was attributed to synergistic effect of $\pi-\pi$ stacking interactions and hydrogen bonding. This bioinspired synergistic toughening strategy opens a new avenue for constructing high performance GO-based nanocomposites in the near future. 


\section{Introduction}

Natural nacre demonstrates extraordinary fracture toughness ranging to 3000 times higher than the intrinsic toughness of its constituent of $\mathrm{CaCO}_{3}$ platelets. This level of toughness is attributed to the interfacial interactions between organic protein matrix and $\mathrm{CaCO}_{3}$ platelets [1-3]. Inspired by this relationship of extraordinary toughness and abundant interface interactions, a range of bioinspired nanocomposites based on different inorganic platelets have been demonstrated, including flattened double-walled carbon nanotubes [4], man-made $\mathrm{CaCO}_{3}$ [5-6], nanoclay [7-8], $\mathrm{Al}_{2} \mathrm{O}_{3}$ flakes [9-10], and layered double hydroxides [11]. Graphene oxide (GO) nanosheets with numerous oxygen-containing groups are an ideal candidate for achieving the artificial nacre construction through the design of different interface interactions, including hydrogen bonding, ionic bonding, $\pi-\pi$ interaction, and covalent bonding. Many GO-based artificial nacre with excellent mechanical properties have been produced by constructing different interface interactions [12-18], for example, the GO-Borate, $r$ GO-PCDO, PGO-PDA, $r$ GO-SL, and $r$ GO-PAPB. In our previous report $[16,17]$, we demonstrated ultra-tough GO-based artificial nacre with long linear molecules of PCDO, which absorbs much energy when the coiled structure is stretched under loading. However, constructing much tougher GO-based artificial nacre remains a great challenge.

Recently, our group has developed a new route to toughen brittle epoxy thermosets with the block ionomer, sulfonated polystyrene-block-poly(ethylene-co-butylene)-blockpolystyrene (SSEBS) via sulfonating polystyrene-block-poly(ethylene-co-butylene)block-polystyrene (SEBS) with 67 wt.\% of polystyrene (PS) [19,20]. Moreover, styrene segments in SEBS can form strong $\pi-\pi$ stacking [21] whereas sulfoacid groups can form 
hydrogen bonds with oxygen-containing groups on the GO surface, which can guarantee strong interfaces between GO and SSEBS and thus facilitate the stress transfer and energy dissipation upon external load.

In this work, we have demonstrated super-tough GO-based artificial nacre through synergistic toughening of $\pi-\pi$ interaction and hydrogen bonding. A new water-soluble long chain molecule, sulfonated styrene-ethylene/butylene-styrene (SSEBS), with many benzene groups was synthesized through sulfonating SEBS triblock copolymers. Then the SSEBS and GO nanosheets were assembled into artificial nacre via vacuum assistedfiltration. The strong $\pi-\pi$ interaction is formed between the benzene groups with GO nanosheets, and hydrogen bonding network is formed between hydroxyl groups, carboxyl groups of GO nanosheets, and sulfonic groups of SSEBS [19-21] as well. The resultant synergistic effect of $\pi-\pi$ interactions and hydrogen bonding improves the efficiency of stress transfer, and long soft SSEBS segments easily deform and extend under loading, dissipating much more fracture energy. The toughness of GO-SSEBS reaches as high as $15.3 \mathrm{MJ} / \mathrm{m}^{3}$, which is eight times higher than that of natural nacre [22], and superior to other GO-based artificial nacre. This bioinspired synergistic toughening of $\pi-\pi$ interactions and hydrogen bonding strategy opens a new avenue for constructing high performance GO-based nanocomposites in the near future.

\section{Experimental section}

\subsection{Materials}

Graphene oxide (GO) was prepared according to our previous work [23]. Detailed preparation process of sulfonated styrene-ethylene/butylene-styrene (SSEBS) is provided in Supporting Information. 


\subsection{Fabrication of SSEBS-toughened GO-based artificial nacre}

Firstly, $1.5 \mathrm{~g}$ of each polymer (GO, SSEBS) was dispersed in $1000 \mathrm{ml}$ deionized water with the aid of sonication for $30 \mathrm{~min}$, and then stirring for $3 \mathrm{~h}$ and to prepare $1.5 \mathrm{mg} / \mathrm{ml}$ aqueous solutions of GO, and SSEBS, respectively. Then, a certain amount of SSEBS solution was added into the GO solution according to desired mass ratios. The mixtures were then stirred for $12 \mathrm{~h}$ to form a homogeneous solution. Finally, the SSEBSintercalated GO-based artificial nacre was obtained with vacuum-assisted filtration selfassembly process. Additionally, the GO-PVA film was prepared using the same preparation protocol to GO-SSEBS but according to the ratio, $50 \mathrm{wt} \% \mathrm{PVA}$, in previous reports as a comparison to evaluate the advantage of SSEBS over PVA as the intercalating agent. Detailed formulations and sample designation are shown in Table 1.

\subsection{Characterization}

Typical tapping-mode atomic force microscopy (AFM) measurements were performed on a Bruker Multi Mode 8 SPM instrument (USA). Scanning electron microscopy (SEM) images were obtained using a field-emission scanning electron microscope (FEI-SEM S4800) at an acceleration voltage of $5 \mathrm{kV}$. The accelerating voltage was increased up to $15 \mathrm{kV}$ for energy dispersive X-ray spectroscopy (EDX) measurements. XPS measurements were performed using ESCAlab 250XI electron spectrometer. X-ray diffraction (XRD) tests were carried out using a Rigaku X-ray generator $\left(\mathrm{Cu} \mathrm{K}_{\alpha}\right.$ radiation). Infrared (IR) spectra of all films were recorded on a Bruker Vetex-70 IR spectrometer (Germany) using an attenuated total reflectance (ATR) mode. Raman spectra were obtained with an ALMEGA-Dispersive Raman (Thermo Nicolet) with 514.5 $\mathrm{nm}$ excitation. Thermogravimetric analysis (TGA) was conducted on a TA Q50 thermal 
analyzer (TGA, USA) in a temperature range of 50 to $750^{\circ} \mathrm{C}$ in nitrogen with a heating rate of $5{ }^{\circ} \mathrm{C} / \mathrm{min}$. Mechanical properties were measured using an Instron $30 \mathrm{kN}$ tensile tester at $23 \pm 2{ }^{\circ} \mathrm{C}$ at a loading rate of $1 \mathrm{~mm} / \mathrm{min}$ with a gauge length of $10 \mathrm{~mm}$. All of the samples were cut into strips with a length of $40 \mathrm{~mm}$ and a width of $2 \mathrm{~mm}$ width before measurement. The results for each sample ware based on the average value of at least 5 specimens.

\section{Results and discussion}

The size of GO nanosheets is in the range of $1.0-5.0 \mu \mathrm{m}$ with a thickness of $\sim 1 \mathrm{~nm}$ (Figure S1), and the water-soluble SSEBS was synthesized via sulfonating SEBS (Scheme S1). After sulfonation, four new absorption peaks appear in the infrared spectrum (IR), respectively locating at $3397 \mathrm{~cm}^{-1}$ (the stretching vibration of O-H in $\mathrm{SO}_{3} \mathrm{H}$ ), $1126 \mathrm{~cm}^{-1}, 1032 \mathrm{~cm}^{-1}$ and $1003 \mathrm{~cm}^{-1}$ (stretching vibration of S-O) [24,25] (Figure S2A). Elemental analysis results show that SSEBS contains $5.14 \mathrm{wt} \% \mathrm{~S}$ element, and the aqueous solution of $5.0 \mathrm{wt} \%$ SSEBS looks brown and transparent (Figure S2B), revealing the water-solubility of SSEBS. The GO-SSEBS artificial nacre is assembled via vacuumassisted filtration, as shown in Figure 1a. $1.0 \mathrm{wt} \%$ GO suspension was prepared by dispersing the GO nanosheets into deionized water with the aid of sonication and stirring, and then the $1.0 \mathrm{wt} \%$ aqueous solution of SSEBS was added. The mixture was further stirred for 12 hours, and filtrated with vacuum-assistance. Different SSEBS content of resultant artificial nacre were fabricated for comparison, and the resultant artificial nacre were designated as GO-S-n, in which n represents the SSEBS content. In this study, five kinds of artificial nacre are fabricated, listed as: GO-S-2, GO-S-5, GO-S-10, GO-S-15, and GO-S-20. The real content of SSEBS is further confirmed, as shown in Fig. S5 and 
listed in Table S1. The digital photograph of flexible GO-S-10 is shown in Figure $1 \mathrm{~b}$. Cross-section morphology of GO-S-10 indicates clearly well orderly nacre-mimetic structure, as shown in Fig.1c. The elemental map reveals uniform dispersion of presence of $\mathrm{C}, \mathrm{O}$ and $\mathrm{S}(2.38 \mathrm{wt} \%)$ elements, as shown in Figs. 1d-e.

To further verify the presence of SSEBS in the gallery of adjacent GO nanosheets, Xray photoelectron spectroscopy (XPS) tests were conducted. Besides typical binding energy peaks for pure GO (Fig. S3), a new peak at $286.9 \mathrm{eV}$ belonging to the binding energy of C-S bonds appears in the C1s spectrum of GO-S-10, as shown in Fig. 2a, and the content of S element was determined to be $2.42 \mathrm{wt} \%$ (Fig. S4). Raman spectra demonstrate that the $I_{\mathrm{D}} / I_{\mathrm{G}}$ ratio value decreases from 1.39 for pure GO to 1.31 for GO-S10, as shown in Figure 2b. Moreover, though pristine SSEBS only exhibits a band at $1093 \mathrm{~cm}^{-1}$, adding SSEBS makes $G$ band $\left(1593 \mathrm{~cm}^{-1}\right)$ for pure GO shift to higher wavenumbers $\left(\sim 1599 \mathrm{~cm}^{-1}\right)$, mainly because of the strong $\pi-\pi$ interactions between GO nanosheets and SSEBS[21]. In comparison, introducing PVA causes $G$ and $D$ bands to shift to lower wave-numbers (1588 and $\left.1346 \mathrm{~cm}^{-1}\right)$. In addition, two new absorption peaks at 2930 and $2856 \mathrm{~cm}^{-1}$ (stretching vibrations of $\mathrm{CH}_{2}$ in SSEBS chains) appear in the IR spectrum of GO-S-10, further indicating the presence of SSEBS in the gallery of adjacent GO nanosheets (Figure 2c).

$\mathrm{X}$-ray diffraction (XRD) measurements were carried out to determine the interlayer distance ( $d$-spacing) of the resultant the interlayer distance artificial nacres. As shown in Fig. 2d and Table S1, pure GO film displays a small $d$-spacing of $7.21 \AA$. After introducing SSEBS, the $d$-spacing value of artificial nacre steadily increases with increasing polymer loading level, for instance, a $d$-spacing of $7.34 \AA$ for GO-S-2, $7.72 \AA$ 
for GO-S-5, $8.85 \AA$ for GO-S-10, $9.01 \AA$ for GO-S-15, and $9.27 \AA$ for GO-S-20. The gradually increasing $d$-spacing strongly further indicates the successful intercalating of SSEBS into gallery of the adjacent GO nanosheets. Moreover, upon the input SSEBS content above $10 \mathrm{wt} \%$, the diffraction peak at about $19.3^{\circ}$ corresponding to characteristic peak of SSEBS appears.

Typical stress-strain curves of GO-S-n artificial nacre are shown in Fig. 3a. The presence of small amount of SSEBS causes GO nanosheets to exhibit remarkably enhanced tensile strength and extensibility due to strong interfacial interactions between GO nanosheets and SSEBS as well as high deformation capabilities of SSEBS. As shown in curve 1 of Fig. 3a, the pure GO film displays a tensile strength of about $90 \mathrm{MPa}$ and toughness of $1.53 \mathrm{MJ} / \mathrm{m}^{3}$, consistent with previous reports [16-18]. The pure SSEBS film exhibits a much lower tensile strength of about $14 \mathrm{MPa}$, but a much higher strain of $100 \%$ (Table S1).

The tensile strength and toughness of GO-S-n artificial nacre, steadily increase with increasing SSEBS content, and reach the maximum values when the SSEBS content is about $10 \mathrm{wt} \%$ (GO-S-10). The tensile strength and toughness of GO-S-10 reach up to 158 $\pm 6.0 \mathrm{MPa}$ and $15.3 \pm 1.5 \mathrm{MJ} / \mathrm{m}^{3}$, corresponding to $76 \%$ and $900 \%$ improvement compared to that of pure GO film, respectively. However, when the SSEBS content further increases, the tensile strength and toughness dramatically decreases, as shown in Fig. 3b-c. When the SSEBS content is lower than $10 \mathrm{wt} \%$, the presence of SSEBS cannot provide enough space for the mutual slippage of GO nanosheets, which limits the dissipation of energy in process of fracturing of $\pi-\pi$ interactions, deforming and extending of SSEBS under loading. However, when the SSEBS content is higher than 10 
wt $\%$, the excess SSEBS probably only acts as the intercalating impurity to increase both the interlayer spacing and the area of cross-section of GO-S-n artificial nacre. Meanwhile, the excess SSEBS may also hinder the stress transfer between adjacent GO nanosheets, which is also observed by previous report [18]. Detailed mechanical properties of other GO-S-n artificial nacre are listed in Table S1.

Compared to the pure GO film, the artificial nacre of GO-S-10 exhibits a typical stressstrain curve with two failure stages, including the elastic deformation, and hardening stage, as shown in Fig. 3d. This is because that there are numerous interface interactions between GO nanosheets and SSEBS. For example, $\pi-\pi$ interactions are formed between GO nanosheets and long polystyrene (PS) segments in SSEBS, and the hydrogen bonding are formed between sulfonic acid groups on PS segments with abundant hydroxyl, carboxyl groups on the surface of GO nanosheets. The proposed fracture process is illustrated as show in Fig. 3f. Taking the artificial nacre of GO-S-10 as an example, the corresponding stress-strain curve is shown in Fig. 3d. When the loading starts, the GO-S10 artificial nacre first show the plastic deformation, designated as stage I, the soft long aliphatic chains of EB segments in SSEBS start to deform and extend from the randomly coiled conformation between adjacent GO nanosheets, resulting in large strain. This form of plasticization facilitates the mutual slippage of GO nanosheets upon loading, which also results in high dissipation of energy. When continuously stretching, EB chains further extend along the direction of stretching. Then, the stage II of the hardening stage starts, and the EB chains are stretched to uncoil until fracture. In this process, firstly, the hydrogen bonds fracture, and then the $\pi-\pi$ stacking interactions begin to rupture with increasing loading. Finally, a fraction of GO nanosheets are pulled out, as shown in Fig. 
3e. The S element is detected on the pulled out GO nanosheets, indicating that SSEBS is also pulled out with GO nanosheet in the facture process, mainly because of the strong $\pi$ $\pi$ stacking interactions between SSEBS and GO nanosheets.

This form of artificial nacre shows super high toughness compared to natural nacre and other GO-based nanocomposites, and the comparison of stress strain curves is shown in Fig. 4, with detailed mechanical properties listed in Table S2. The artificial nacre of GOS-10 exhibits toughness of $15.3 \mathrm{MJ} / \mathrm{m}^{3}$, which is almost about 8.5 times of that of natural nacre with $1.8 \mathrm{MJ} / \mathrm{m}^{3}$, and other GO-based nanocomposites with different cross-linking strategies. For example, Tian et al. [26] applied polyetherimide (PEI) cross-link GO nanosheets and achieved strong GO-based nanocomposites (GO-PEI) with tensile strength of $\sim 209.9 \mathrm{MPa}$, but low toughness of $0.23 \mathrm{MJ} / \mathrm{m}^{3}$, and the corresponding stressstrain curve is shown in curve 10 of Fig. 4. This behavior is because of the strong covalent bonding, which limits the mutual slippage of adjacent GO nanosheets resulting in high strength but low toughness. The other GO-based nanocomposites with covalent bonding, such as GO-PAA, GO-GA, GO-PDA, GO-Borate, and GO-PCDO, also demonstrate the high tensile strength but low toughness. For GO-based nanocomposites with ionic bonding, such as GO- $\mathrm{Mg}^{2+}$ and GO-Ca ${ }^{2+}$, the enhancements in toughness and strength are also difficult. On the other hand, although the tensile strength of polymer reinforced GO nanosheets nanocomposites show high strength, they exhibit low toughness because of weak interactions, such as GO-PMMA, GO-PVA, GO-MoS ${ }_{2}$-TPU, and $\mathrm{GO}-\mathrm{Al}_{2} \mathrm{O}_{3}-\mathrm{PVA}$. Recently, the high toughness graphene-based nanocomposites are achieved through chemically reducing the GO-based nanocomposites, for example, GO- 
CS, GO-CNC, and GO-HPG. In this study, we focused primarily on the GO-based nanocomposites.

There are two main reasons for the much higher tensile toughness for GO-S-10 as compared with other GO-based artificial nacre. Firstly, the strong $\pi$ - $\pi$ stacking interactions between poly(styrene) segments in SSEBS and GO as well as hydrogen bonding interactions between the sulfoacid groups of SSEBS and oxygen-containing groups on the GO surface guarantee strong their interfacial interactions and the effective stress transfer. In other words, adjacent GO nanosheets in our system are interlocked by the physical interactions ( $\pi-\pi$ stacking and hydrogen bonding), which allows the readily slippage of GO during tension. By contrast, GO-based composites based on chemical bonds including covalent bonds (e.g. GO-GA, GO-PDA, GO-Borate and GO-PCDO) or ionic bonds (GO- $\mathrm{Mg}^{2+}$ and GO-Ca ${ }^{2+}$ ) can restrict the mutual slippage of GO sheets under external tensile load, thus leading to a much low elongation and tensile toughness despite the high tensile strength. Moreover, the long soft EB segment in SSEBS located in the gallery of layered GO materials tend to deform and extend from the randomly coiled conformation upon loaded, which can absorb much energy and also promote the mutual slippage of GO sheet, thus facilitating the dissipation of fracture energy and leading to a noticeable increase in the tensile toughness. As a result, SSEBS-toughed GO-based artificial nacre shows much higher toughness than other GO-based counterparts with similar structure.

\section{Conclusions}

Inspired by natural nacre, we created super-tough GO-based artificial nacre via synergistic interface interactions. The synthesized water-soluble sulfonated SEBS 
copolymer provides numerous functional groups, resulting in formation of $\pi-\pi$ interaction and hydrogen bonding with GO nanosheets. This kind of synergistic interface interactions achieves high toughness of $15.3 \mathrm{MJ} / \mathrm{m}^{3}$, superior to natural nacre and other GO-based nanocomposites. This work provides a novel bioinspired toughening strategy for creating super-tough GO-based nanocomposites.

\section{Acknowledgements}

${ }^{\S} \mathrm{Z}$. Xu is the co-first author and P. Song and Z. Xu contributed equally to the work.

This work was financially supported by the Natural National Science Foundation of China (51522301, 51303162, 51304166 and 51628302) and the Non-profit Project of

Science and Technology Agency of Zhejiang Province of China (Grant No. 2013C32073). P.S. was also supported by an Alfred Deakin Postdoctoral Research Fellowship at Deakin University.

\section{Supplementary data}

Supporting Information: Synthesis of sulfonated SEBS (SSEBS), AFM images of GO, FT-IR spectra of SEBS and SSEBS, C1s spectrum of GO film, XPS survey of GO and GO-S-10, TGA curves of GO, SSEBS and their composites, SEM images of GO, and GO-S-n composites, and mechanical properties of the natural nacre, artificial nacre of the GO-S-n and other GO- based layered materials.

\section{REFERENCES}

[1] Bouville F, Marire E, Meille S, Van de Moortèle B, Stevenson AJ, Deville S. Strong, Tough and Stiff Bio-inspired Ceramics from Britle Constitutes. Nat Mater 2014; 13: 508-514. 
[2] Huang Z, Li X. Order-Disorder Transition of Aragonite Nanoparticles in Nacre. Phys Rev Lett 2012; 109: 025501.

[3] Wang J, Cheng Q, Tang Z. Layered Nanocomposites Inspired by the Structure and Mechanical Properties of Nacre. Chem Soc Rev 2012; 41: 1111-1129.

[4] Cheng QF, Li M, Jiang L, Tang ZY. Bioinspired Layered Composites Based on Flattened Double-Walled Carbon Nanotubes. Adv Mater 2012; 24: 1838-1843.

[5] Finnemore A, Cunha P, Shean T, Vignolini S, Guldin S, Oyen M, Steiner U. Biomimetic Layer-by-Layer Assembly of Artifical Nacre. Nat Commun 2012; 3: 966.

[6] Li XQ, Zeng HC. Calcium Carbonate Nanotablets: Bridging Artifical to Natural Nacre. Adv Mater 2012; 24: 6227-6282.

[7] Tang Z, Kotov NA, Magonov S, Ozturk B. Nanostructured Artifical Nacre. Nat Mater 2003; 2: 413-418.

[8] Podisiadlo P, Kaushik AK, Arruda EM, Waas AM, Shim BS, Xu JD.; Nandivada H, Pumpplin BG, Lahann J, Ramamoorthy A, et al. Ultrastrong and Stiff Layed Polymer Nanocomposites. Science 2007; 318: 80-83.

[9] Bonderer LJ, Studart AR, Gauckler LJ. Bioinspired Design and Assembly of Platelet Reinforced Polymer Film. Science 2008; 319: 1069-1073.

[10] Deville S, Saiz E, Nalla RK, Tomsia AP. Freezing as a Path to Build Complex Composites. Science 2006; 311: 515-518.

[11] Yao HB, Fang HY, Tan ZH, Wu LH, Yu SH. Biologically Inspired, Strong, Transparent, and Functional Layed Organic-Inorganic Hybrid Films. Angew Chem Int Ed 2010; 49: 2140-2145. 
[12] Cheng QF, Jiang L, Tang ZY. Bioinspired Layed Materials with Superior Mechanical Performance. Acc Chem Res 2014; 47: 1256-1266.

[13] Huang X, Qi X, Boey F, Zhang H. Graphene-Based Composites. Chem Soc Rev 2012; 41: 666-686.

[14] Dikin DA, Stankovih S, Zimney EJ, Piner RD, Dommett GH, Evmenenko G, Nguyen ST, Ruoff RS. Preparation and Characterization of Graphene Oxide Paper. Nature 2007; 448: 457-460.

[15] Li D, Kaner RB.Graphene-Based Materials. Science 2008; 320: 1170-1171.

[16] Cheng QF, Wu MX, Li MZ, Jiang L, Tang ZY. Ultratough Artifical Nacre Based on Conjugated Cross-linked Graphene Oxide. Angew Chem Int Ed 2013; 52: 3750-3755.

[17] Wan SJ, Li YC, Peng JS, Hu H, Cheng QF, Jiang L. Synergistic Toughnening of Graphene Oxide-Molybdenum Disulfide-Thermoplastic Polyurethane Ternary Artifical Nacre. ACS Nano 2015; 9: 708-714.

[18] Cui W, Li MZ, Liu JY, Wang B, Zhang C, Jiang L, Cheng QF. A Strong Integrated Strength and Toughness Artifical Nacre Based on Dopamine Cross-linked Graphene Oxide. ACS Nano 2014; 8: 9511-9517.

[19] Wu SY, Guo QP, Kraska M, Stühn B, Mai Y-W. Toughening Epoxy Thermosets with Block Ionomers: The Role of Phase Domain Size. Macromolecules 2013; 46: 8190-202.

[20] Wu SY, Guo QP, Peng SH, Hameed N, Karaska M, Stuhn B, Mai YW. Toughening Epoxy Thermosets with Block Ionomer Complexs:A Nanostructure-Mechanical Property Correlation. Macromolecules 2012; 45: 3829-3840. 
[21] Shen B, Zhai WT, Chen C, Lu DD, Wang J, Zheng WG. Melt Blending In situ Enhances the Interaction between Polystyrene and Graphene through $\pi$ - $\pi$ Stacking. ACS Appl. Mater. Interfaces 2011; 3: 3013-3109.

[22] Jackson A, Vincent J, Yurner R. The Mechanical Design of Nacre. Proc R Soc B 1988; 234: 415-440.

[23] Song PA, Cao ZH, Cai YZ, Zhao LP, Fang ZP, Fu SY. Fabrication of Exfoliated Graphene-Based Polypropylene Nanocomposites with Enhanced Mechanical and Thermal Properties. Polymer 2011; 52: 4001-4010.

[24] Weiss RA, Sen A, Willis CL, Pottick LA. Block Copolymer Ionomer.1. Synthesis and Physical Properties of Sulfonated Poly(styrene Ethylene/Butylene Styrene). Polymer 1991; 32: 1867-1874.

[25] Wu SY, Peng SH, Hameed N, Guo QP, Mai YW. A New Route to Nanostructured Thermosets with Block Ionomer Complexs. Soft Matter 2012; 8: 688-698.

[26] Tian Y, Cao YW, Wang Y, Yang WL, Feng JC. Realizing Ultrahigh Modulus and High Strength of Macroscopic Graphene Oxide Papers Through Crosslinking of Mussel-Inspired Polymers. Adv Mater 2013; 25: 2980-2983.

[27] Park S, Lee KS, Bozoklu G, Cai W, Nguyen ST, Ruoff RS. Graphene Oxide Papers Modified by Divalent Ions-Enhancing Mechanical Properties via Chemical Crosslinking. ACS Nano 2008; 2: 572-578.

[28] Park S, Dikin DA, Nguyen ST, Ruoff RS. Graphene Oxide Sheets Chemically Cross-linked by Polyallylamine. J Phys Chem C 2009;113: 15801-15804. 
[29] Li YQ, Yu T, Yang TY, Zheng LX, Liao K. Bio-inspired Nacre-like Composite Films Based on Graphene with Superior Mechanical, Ellectric, and Biocompatible Properties. Adv Mater 2012; 24: 3426-3431.

[30] Hu KS, Tolentino LS, Kulkarni DD, Ye CH, Kumar S, Tsukruk VV. Written-in Coductive Patterns on Robust Graphene Oxide Biopaper by Electrochemical Microstamping. Angew Chem Int Ed 2013; 52: 13784-13788.

[31] Gao Y, Liu YQ, Zu SZ, Peng K, Zhou D, Han BH, Zhang Z. The Effect of Interlayer Adhesion on The Mechanical Behaviors of Macroscopic Graphene Oxide Papers. ACS Nano 2011; 5: 2134-2141.

[32] Putz KW, Compton OC, Palmeric MJ, Nguyen ST, Brinson LC. High-NanofillerContent Graphene-Polymer Nanocomposites via Vacuum-Assisted Self-Assembly. Adv Funct Mater 2012; 24: 3426-3431.

[33] An Z, Compton OC, Putz KW, Brinson LC, Nguyen ST. Bio-Inspired Borate Crosslinking in Ultra-Stiff Graphene Oxide Thin Films. Adv Mater 2011; 23: 2980-2983.

[34] Wang JR, Qiao JL, Wang JF, Zhu Y, Jiang L. Bioinspired Hierarchical AluminaGraphene Oxide-Poly(vinyl alcohol) Artificial Nacre with Optimized Strength and Toughness. ACS Appl Mater Interfaces 2015; 7: 9281-9286. 


\section{Captions of Figures}

Fig. 1. (a) Schematic representation of the fabrication process of GO-S-n artificial nacre via vacuum-assisted filtration. (b) Digital photograph and (c) Cross-section morphology of GO-S-10. (d) Elemental maps and (e) Elemental composition of GO-S-10.

Fig. 2. (a) C1s spectrum of GO-S-10, (b) Raman spectra of SSEBS, pure GO, GO-S-10, and GO-S-50. Appearance of binding energy peaks at 232.7eV (S2s) and $168.9 \mathrm{eV}$ (S2p) together with the peak belonging to C-S (286.8 eV) suggests SSEBS was successfully introduced into GO galleries. (c) FTIR spectra of pure GO, GO-S-10 and SSEBS. (d) XRD spectra of pure GO, GO-S-2, GO-S-5, GO-S-10, GO-S-15, and GO-S-20, respectively.

Fig. 3. (a) Typical stress-strain curves of pure GO film (curve 1), GO-S-5 (curve 2), GOS-10 (curve 3), and GO-S-15 (curve 4). (b) Tensile strength and (c) toughness of GO and GO-S-n artificial nacre with different SSEBS content, indicating that both tensile strength and toughness reach the maximum value at a SSEBS content of about $10 \mathrm{wt} \%$. (d) Typical stress-strain curve of the pure SSEBS film and GO-S-10 artificial. (e) Fracture morphology of GO-S-10 artificial nacre after tensile testing. (f) Proposed fracture mechanism of GO-S-10 artificial nacre.

Fig. 4. Comparison of stress-strain curves with GO-S-10 artificial nacre (Curve 15) with other GO-based nanocomposites, such as GO- $\mathrm{Mg}^{2+}$ (Curve 2) [27], GO-Ca ${ }^{2+}$ (Curve 3) [27], GO-PAA (Curve 4) [28], GO-PVA (Curve 5) [29], GO-PCDO (Curve 6) [16], GOSF (Curve 7) [30], GO-GA (Curve 8) [31], GO-PEI (Curve 9) [26], GO-PMMA (Curve 
10) [32], GO-PDA (Curve 11) [18], GO-Borate (Curve 12) [33], GO-MoS 2 -TPU (Curve 13) [17], and $\mathrm{GO}-\mathrm{Al}_{2} \mathrm{O}_{3}-\mathrm{PVA}$ (Curve 14) [34], respectively. 
Fig. 1
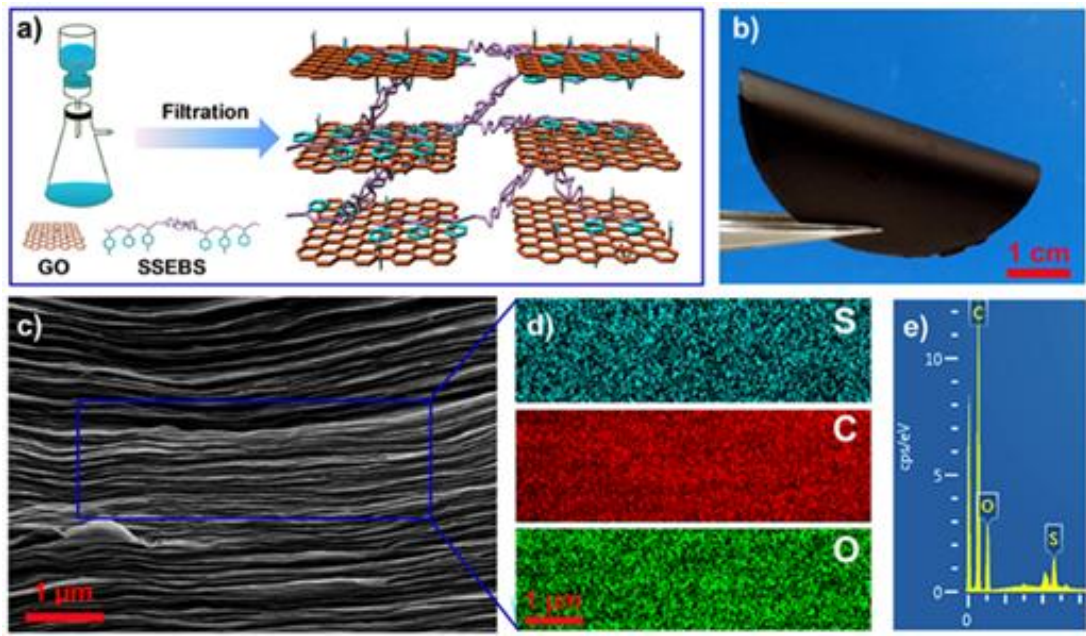

Fig. 2
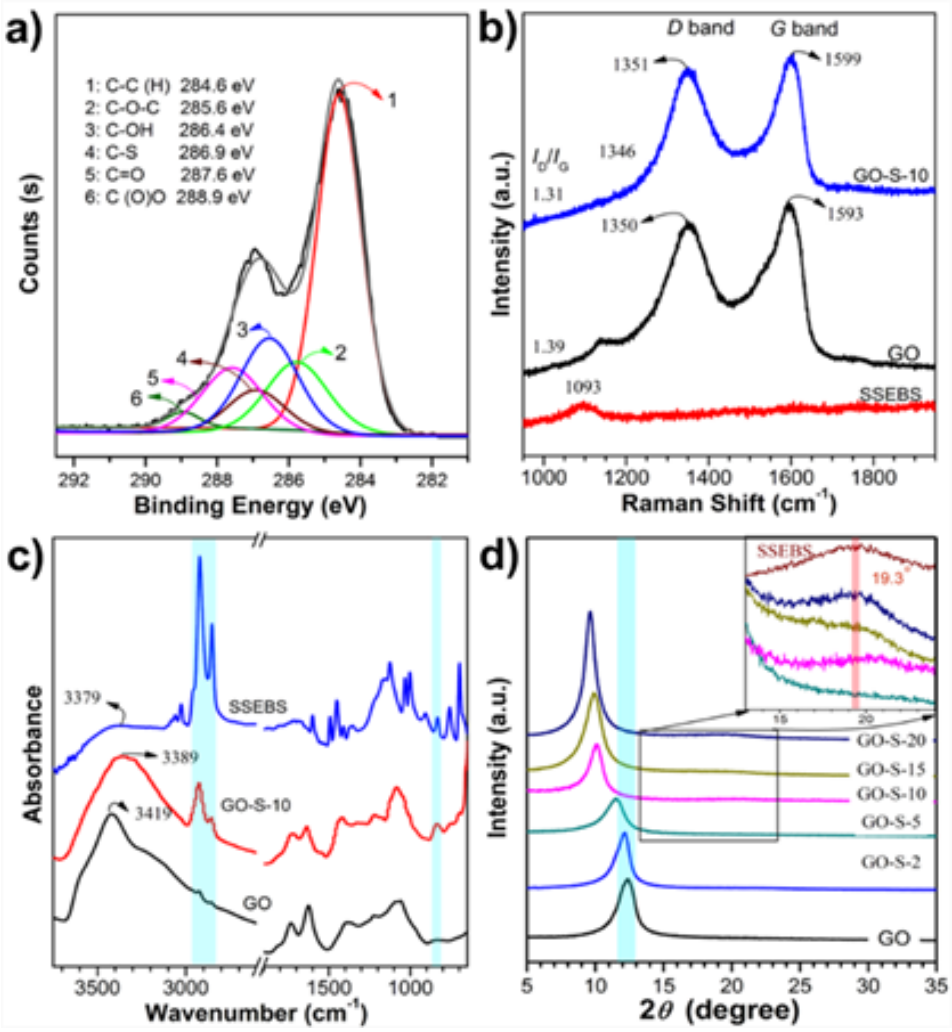
Fig. 3
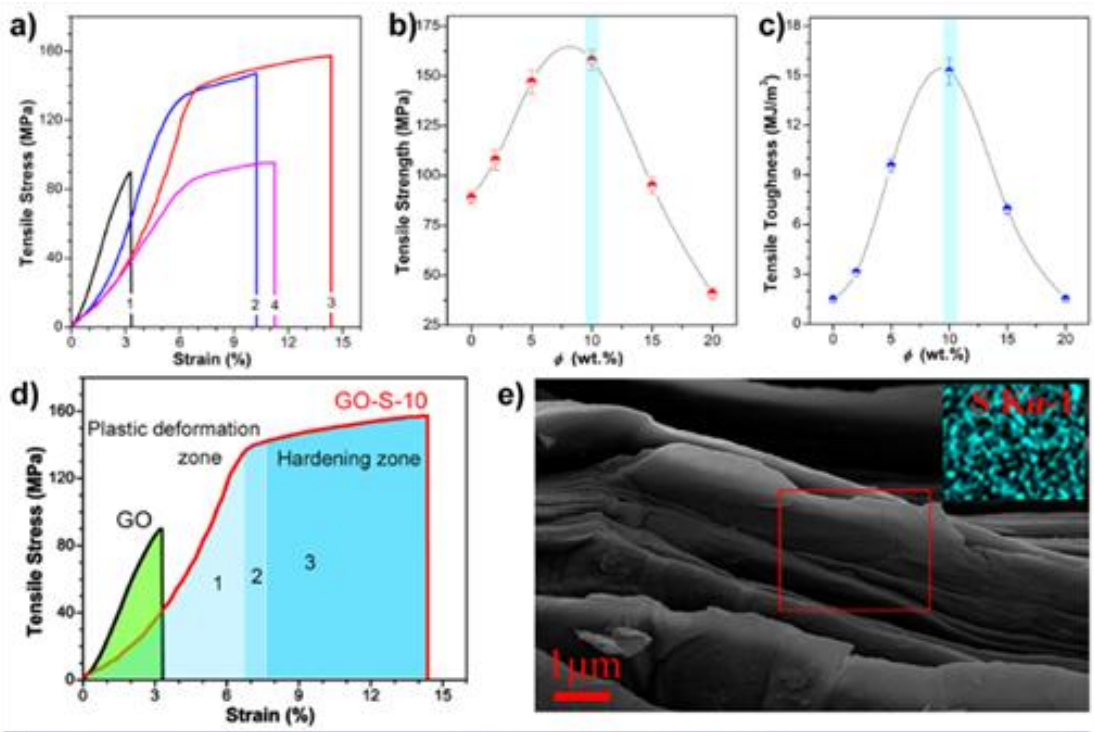

e)
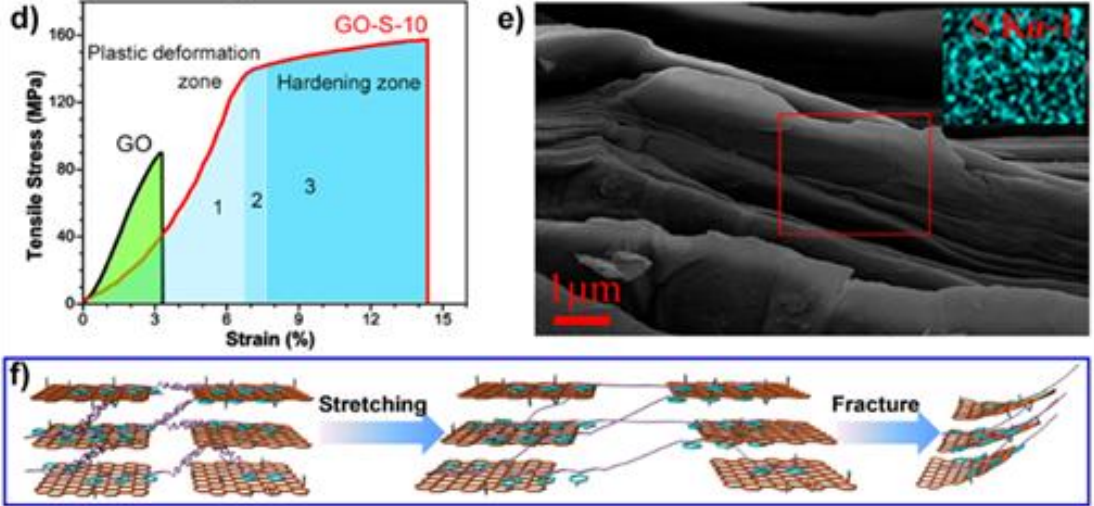

Fig. 4

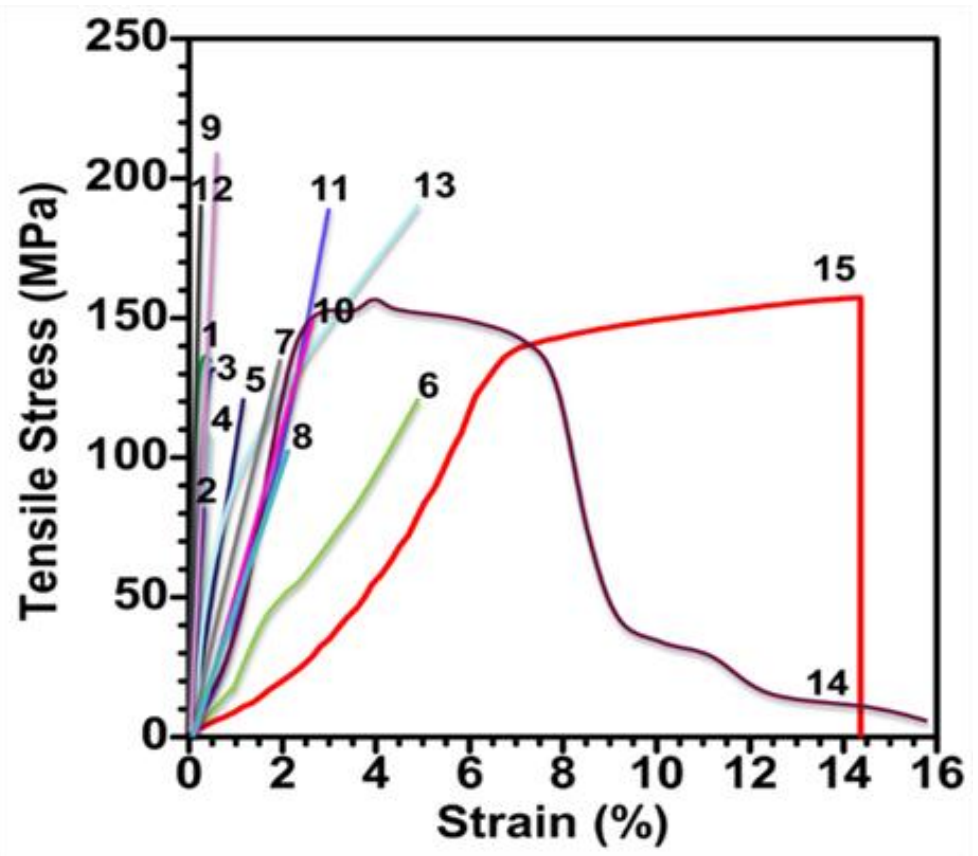


Table 1 Loading level of polymers and $d$-spacing for as-prepared artificial nacre.

\begin{tabular}{lccc}
\hline \multirow{2}{*}{ Run } & \multicolumn{2}{c}{ Loading level of polymer (wt $\%)$} & \multirow{2}{c}{$\begin{array}{c}\text {-spacing }(\AA) \\
\text { by XRD }\end{array}$} \\
\cline { 2 - 3 } & Input & by TGA & \\
\hline GO & - & - & 7.21 \\
GO-S-2 & 2.0 & 1.90 & 7.34 \\
GO-S-5 & 5.0 & 4.90 & 7.72 \\
GO-S-10 & 10 & 9.90 & 8.85 \\
GO-S-15 & 15 & 14.8 & 9.01 \\
GO-S-20 & 20 & 19.7 & 9.27 \\
\hline
\end{tabular}

